CARNETS DE Carnets de géographes

GÉOGRAPHES.

4 | 2012

Géographies critiques

\title{
Des émigrants dans le passage
}

Une approche géographique de la condition de clandestin à Cherbourg et sur les côtes de la Manche

\section{Olivier Thomas}

\section{(2) OpenEdition}

\section{Journals}

Édition électronique

URL : http://journals.openedition.org/cdg/1039

DOI : $10.4000 /$ cdg. 1039

ISSN : 2107-7266

Éditeur

UMR 245 - CESSMA

Référence électronique

Olivier Thomas, «Des émigrants dans le passage », Carnets de géographes [En ligne], 4 | 2012, mis en ligne le 01 septembre 2012, consulté le 24 septembre 2020. URL : http://journals.openedition.org/cdg/ 1039 ; DOI : https://doi.org/10.4000/cdg.1039

\section{(c) $\Theta \Theta \Theta$}

La revue Carnets de géographes est mise à disposition selon les termes de la Licence Creative Commons Attribution - Pas d'Utilisation Commerciale - Pas de Modification 4.0 International. 


\section{DES ÉMIGRANTS DANS LE PASSAGE Une approche géographique de la condition de clandestin à Cherbourg et sur les côtes de la Manche}

\section{OLIVIER THOMAS}

Cette recherche apporte une contribution à la compréhension du passage, et plus largement du fait clandestin. La multiplication des camps informels et des squats d'émigrants aux frontières, mais aussi à l'intérieur de l'Europe depuis une vingtaine d'années marque une transformation du rapport à l'étranger dans nos sociétés. La figure de l'immigrant tend à se confondre aujourd'hui avec celle $\mathrm{du}$ «clandestin», et les conditions de séjour des émigrants, qualifiés «d'illégaux», au sein de l'Union européenne sont extrêmement précaires. En France, le long des côtes de la Manche, la plupart d'entre eux vivent sous des tentes et bénéficient du soutien des associations humanitaires qui leur apportent une aide d'urgence. Le déploiement de mesures sécuritaires et policières par l'Etat depuis la fin des années 1990 contribue à maintenir ces migrants à la marge. Le phénomène suscite de nombreux débats dans la société. Dès lors, il apparaît intéressant pour le chercheur en géographie sociale d'explorer les problématiques qui se cristallisent autour des migrants et de témoigner de ce qui est aujourd'hui une question sociale.

\section{Le fait clandestin comme champ de recherche}

Cette thèse propose d'étudier la géographie actuelle du fait clandestin, entendu comme l'ensemble des problématiques qui peuvent être attachées aux déplacements clandestins d'émigrants et à leurs inscriptions dans les sociétés. Cette géographie, structurée par le voyage clandestin, constitue selon nous la dimension spatiale d'un rapport social entre les émigrants et la société.

\section{Le passage comme objet d'étude}

Le voyage clandestin ne peut se penser seul, mais nécessairement en rapport avec la population qui l'anime. Toutes les personnes qui transitent par les lieux d'installation informels le long du littoral de la Manche ont en commun d'être dans le voyage, et ce quelle que soit leur nationalité (Irakiens, Iraniens, Erythréens, Ethiopiens, des Palestiniens ou encore des Vietnamiens). Elles ont quitté leur pays d'origine et tentent de rejoindre un pays de destination (l'Angleterre, l'Irlande, mais aussi le Danemark, la Norvège ou la Suède). Le passage, c'est cet «entre-deux» qui qualifie un moment entre d'un côté le franchissement de la première frontière (celle du pays d'origine) et de l'autre l'engagement dans des démarches de régularisation (dépôt d'une demande d'asile par exemple). Le passage est aussi un espace. Il désigne l'itinéraire du «clandestin» depuis son pays d'origine jusque dans le pays de destination, ce qui inclut les étapes qui viennent ponctuer son parcours. Le terme de passage désigne donc à la fois le mouvement (d'une société à l'autre) et la présence (dans les sociétés traversées), de sorte que cette notion n'appelle pas uniquement une étude des logiques qui animent les migrations clandestines, mais aussi une approche géographique des rapports entre les «clandestins» et les sociétés.

\section{Les terrains de recherche et la méthode mise en oeuvre}


La recherche privilégie une démarche inductive et s'appuie sur un travail de terrain mené depuis 2008 dans l'agglomération de Cherbourg-Octeville. La réflexion est nourrie de comparaisons dans les villes portuaires Transmanche (Dieppe, Calais, Dunkerque...) et dans la région Nord (St Omer, Norrent-Fontes, Angres, Steenvoorde). Des recherches ont également été menées dans le $10^{\text {ème }}$ arrondissement de Paris pour saisir le rôle de cet espace dans le cadre du voyage clandestin en Europe. La définition des différents terrains relève d'une construction : l'étude de la projection dans l'espace du fait clandestin a permis de développer des connaissances concernant les itinéraires clandestins et la localisation des différents squats et camps informels situés à proximité des points de passage. Les pratiques spatiales quotidiennes des migrants ont aussi été observées lors du travail d'enquête.

L'enquête qualitative mobilise l'observation participante dans le cadre d'un engagement bénévole au sein de l'association Itinérance à Cherbourg. Plus largement, les associations mobilisées autour des émigrants le long du littoral de la Manche et dans le nord de la France ont constitué une entrée importante lors du travail d'observation sur le terrain. La photographie et la tenue d'un journal de terrain ont été des outils majeurs tout au long de la recherche. Une série d'entretiens a aussi été réalisée auprès de différents acteurs concernés par les problématiques attachées à la présence des migrants «clandestins». Enfin, un travail de revue de presse a été mené à Cherbourg et un intérêt particulier a été porté à l'actualité médiatique du thème des clandestins.

En complément du travail bibliographique dans le champ scientifique, les oeuvres produites dans les champs artistiques et culturels ont fait l'objet d'une attention particulière (cinéma, littérature, théâtre, photographie). Elles constituent des témoignages et participent à mettre en débat la question sociale étudiée. Mobilisées comme des outils pour stimuler la réflexion, l'utilisation de ces sources est aussi l'occasion pour le chercheur de déplacer le regard et de développer une sensibilité et une acuité particulières vis-à-vis de l'objet d'étude.

Tout au long du travail de recherche, l'étude de la condition de clandestin a été le moyen de saisir les rapports des migrants avec la société à l'échelle locale, pour apporter des éléments de connaissances sur le passage, ainsi que pour contribuer, dans une perspective plus large, à la compréhension des sociétés par l'espace.

\section{Apports d'une approche géographique de la condition de «clandestin»}

L'étude de la condition de «clandestin» réalisée dans le cadre de cette thèse permet de porter un regard sur le fait clandestin, d'une part en questionnant le caractère contraint des mobilités des émigrants dans le passage, et d'autre part en s'interrogeant sur la place des migrants «clandestins» dans la société. Il s'agit de deux apports majeurs de cette recherche.

Les mobilités des migrants ne sont pas uniquement le produit de logiques propres au mouvement d'émigration, elles relèvent également des rapports entre les migrants et les sociétés traversées. Dès lors, qu'il s'agisse de l'itinéraire depuis le pays d'origine ou des pratiques spatiales quotidiennes, les mobilités des émigrants dans le passage apparaissent, par leur caractère contraint, comme l'indicateur d'un rapport social entre les sociétés européennes et les «clandestins». En effet, la mobilité s'inscrit aujourd'hui dans nos sociétés comme un attribut - voire une norme - qui contribue à définir et à valoriser les positions sociales les plus élevées. La possibilité de cette mobilité est aujourd'hui refusée à la majorité des émigrants qui se rendent en Europe et tentent de s'y déplacer.

La société est, de fait, confrontée à un phénomène nouveau d'émigration qui vient bousculer ses rapports à l'étranger : quelle place accorder aux émigrants qui se déplacent clandestinement ? Les réponses apportées aujourd'hui sont contradictoires. Elles opposent une forme de gestion administrative et policière du fait clandestin à une autre forme de gestion, d'ordre humanitaire et 
sociale, émanant de la société locale (bénévoles, militants, élus). A Cherbourg, comme dans l'ensemble des camps ou des squats recensés le long du littoral de la Manche, l'étude de l'intégration politique du phénomène du passage révèle cette tension entre "prise en charge» des émigrants et «mise à distance». Près de dix ans après la fermeture du centre de Sangatte, les lieux d'installation informels des "clandestins», loin d'avoir disparus, ont été relégués dans des espaces de marge. Qu'il s'agisse de rendre invisible les migrants et/ou leurs conditions d'accueil déplorables, la géographie actuelle du fait clandestin sur le littoral est à lire comme la projection spatiale d'un rapport social entre les émigrants et la société. Elle témoigne de la place accordée à «l'autre» dans la société.

Fiche informative

Lien électronique pour télécharger la thèse

http://halshs.archives-ouvertes.fr/SHS/tel-00673859/fr/

\section{Discipline}

Géographie

\section{Directeur}

Benoît Raoulx

\section{Université}

Université de Caen

Membres du jury de thèse, soutenue le 30 novembre 2009

-Christian PIHET, Professeur de géographie à l'Université d'Angers

-Claudio CERRETI, Professeur de géographie à l'Università degli studi di Roma Tre

-Djemila ZENEIDI, Chargée de recherches CNRS à l'Université de Bordeaux III

-Thierry BULOT, Professeur de sociolinguistique urbaine à l'Université de Rennes II

-Patrice CARO, Professeur de géographie à l'Université de Caen Basse-Normandie

-Benoît RAOULX, Maître de Conférences, Habilité à Diriger les Recherches, à l'Université de Caen Basse-Normandie

\section{Situation professionnelle actuelle}

Membre associé de l'UMR ESO 6590 CNRS

\section{Contact de l'auteur}

\section{olivier.thomas@unicaen.fr}

